\title{
DIFFICULT WEANING AFTER PULMONARY ENDARTERECTOMY FOR CHRONIC PULMONARY EMBOLISM: A CASE REPORT.
}

Melchisedec, Sheetal Garg, H. S. Bedi ,Valsa Verghese, Anish. G. Varghese

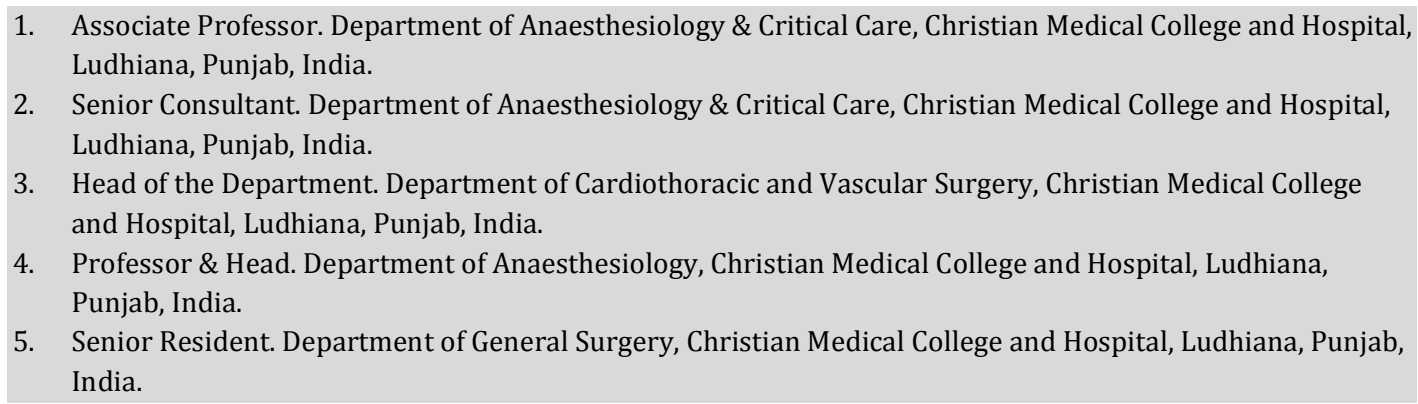

\section{CORRESPONDING AUTHOR:}

Dr. Melchisedec,

Associate Professor,

Department of Anaesthesiology and Critical Care,

Christian Medical College and Hospital,

Ludhiana, Punjab, India-141008.

E-mail: drmelchisedec@yahoo.com

KEYWORDS: Chronic pulmonary thromboembolism, pulmonary embolism, pulmonary endarterectomy, anaesthesia.

SUMMARY: A 33 yrs old male presented with dyspnea on exertion since 6yrs was diagnosed to have chronic thromboembolic pulmonary hypertension (CTEPH) for which he underwent pulmonary endarterectomy (PEA) using deep hypothermic circulatory arrest (DHCA). After the operation the patient developed reperfusion edema and hypoxaemia because of which patient had delayed weaning from mechanical ventilation.

INTRODUCTION: PEA is regarded as a promising and a potentially curative surgical procedure for CTEPH. Post-operative management for PEA is very challenging as it involves the management of intrapulmonary shunting with desaturation as a result of reperfusion pulmonary edema which can lead to prolonged post-operative mechanical ventilation and is an important cause of post-operative mortality and morbidity.

CASE REPORT : A 33 year old male a school teacher by profession, a non smoker and non alcoholic, not a known diabetic or hypertensive, was admitted with complaints of mild cough and dyspnea on exertion since 5 years and worsening of symptoms since six months. General Physical examination revealed an anxious patient with Respiratory rate (RR) 30/min, Pulse rate (PR) 90/min, Blood Pressure (BP) 100/70 mmHg and Oxygen saturation (Spo2) at room air was $78 \%$.

There were no signs of deep venous thrombosis. His Chest X-ray (CXR ) showed increased bronchovascular markings .Electrocardiograph ( ECG) showed a P-Pulmonale, T wave inversion in V1 -V5 and Right Ventricular Hypertrophy (RVH). Transthoracic echocardiography (TTE) revealed a dilated right atrium/right ventricle (RA/RV), mild mitral regurgitation/tricuspid regurgitation( MR/TR), Right ventricular dysfunction (RVEF 20\%), 
Normal LV function (LVEF 55\%) and Pulmonary artery systolic pressure (PASP) of 90mmHg. Venous doppler ultrasound of the Lower limbs showed no evidence of deep vein thrombosis( DVT).Pulmonary function tests (PFT) showed a moderate restriction with ventilatory defect. His Baseline arterial blood gases (ABG) showed a Ph:7.48, Pao2: 46.0, Pco2 : 32.0, ABE : 3.8 . Computed tomography pulmonary angiography( CTPA) revealed a thrombus in the right main pulmonary artery (MPA) [figure1]. His lab investigations showed a hemoglobin( $\mathrm{Hb}$ ) of $18 \mathrm{gm} \%$, Negative D-dimer test, and a Negative thrombotic screen (LA / PNH negative).

The patient was started on treatment with Tab Nicoumalone (acitrom) $2 \mathrm{mg}$, Tab Bosentan 125 mg OD, Tab Tadalafil 20mg OD, and Tab Torsemide 10 mg OD.

Patient was taken up for PEA under DHCA. Tab Pantoprazole $40 \mathrm{mg}$ was given as premedication the night before the surgery. On reaching the operating room ECG, non invasive blood pressure (NIBP) and Spo2 were connected. Under local anaesthesia radial artery, femoral artery and peripheral venous cannulation were done. Patient was painted and draped and the presence of the operating surgeon and the perfusionist was confirmed prior to induction of general anaesthesia keeping in mind the sudden hemodynamic collapse during the induction period.

Patient was induced with Inj midazolam $2 \mathrm{mg}$, Inj fentanyl 200ug, Inj Thiopentone $200 \mathrm{mg}$, and Inj rocuronium $100 \mathrm{mg}$ iv. Trachea was intubated with Endotracheal tube no:8.5 . After intubation pulmonary artery( $\mathrm{PA}$ ) catheter was inserted via the right internal jugular vein (IJV). PA pressure was 92/38 and arterial blood pressure (ABP) of 97/59. Two units of blood were taken out for autologous transfusion later.

After full heparinization patient was put on cardiopulmonary bypass (CPB). Anaesthesia and surgery proceeded without incident during $\mathrm{CPB}$. Inj methylprednisolone 1gm was given through the bypass prior to DHCA. Patient was gradually cooled to $18^{\circ} \mathrm{C}$. Additional cooling of the head and neck was done by keeping ice packs around the head and neck. Alpha stat strategy was used to maintain intra-op ABG's. Pulmonary arteriotomy was done and embolectomy was done from the right pulmonary artery [figure2]. A total DHCA time of 21 mins were taken to perform the pulmonary endarterectomy.

CPB was restarted after closing the pulmonary artery and the patient was rewarmed slowly keeping a gradient between the perfusate and the patient of $7^{\circ} \mathrm{C}$. The patient was rewarmed up to $36^{\circ} \mathrm{C}$ and was weaned off the bypass once the patients temperature reached $37^{\circ} \mathrm{C}$. Heparin was reversed with an equivalent dose of protamine (1:1). Infusions of Inj milrinone , Inj nitroglycerine, Inj dobutamine, Inj norepinephrine ,was started to support the heart.

Postoperatively the patient was kept on Pressure Control Mode ventilation with $100 \%$ fio 2 and a peep of $10 \mathrm{~cm} \mathrm{H} 2 \mathrm{o}$ as his Pao 2 remained between 46 to $60 \mathrm{mmHg}$ from day 1 to 4 . Post operative CXR showed marked hyperperfusion of the lung on the operated side which cleared over the next 3 days. Inj Furosemide infusion was started at $10 \mathrm{mg} / \mathrm{hr}$. Inj methyl prednisolone was continued for 3 days post op. Inj Heparin infusion was started at 1000 units/hr.

From day 5 to 12, the patients Pao2 kept increasing gradually and weaning from the mechanical ventilation was started as the patient started to maintain a Pao2 of 80 with an fio2 of 50\%. Patient was extubated on day 13. His ABG after extubation showed Ph 7.45, Paco2: 33.3, Pao2: 78.2, BE : 3.2.

After extubation patient was maintained on intermittent BiPAP and 02 via facemask from day 13 to 20. Oxygen saturation remained around 90\% with BiPAP and up to $80 \%$ with oxygen via facemask dropping down to $72 \%$ without oxygen. 
Over the next three days (day 21 to 23) the patient started maintaining an spo2 of $92 \%$ with o 2 via face mask and $90 \%$ without oxygen and remained comfortable without BiPAP. The patient was given intermittent oxygen with face mask and was mobilized during the off oxygen period. He was completely weaned off oxygen therapy by $28^{\text {th }}$ post-op day. Repeat chest x-ray and ABG, on day 30 was within normal limits. The patient was maintaining an Spo 2 of $96 \%$ on room air for two consecutive days prior to discharge. His repeat Echo showed a RVEF of $40 \%$ and PA pressure of $35 \mathrm{mmHg}$. The patient was able to mobilize and communicate comfortably and was discharged from the hospital on $31^{\text {st }}$ post-op day.

On follow up after 7 days of discharge the patient was asymptomatic and was carrying out his routine activities without any discomfort. One month later he was able to perform his duties as a teacher in a school.

DISCUSSION: Chronic thromboembolic pulmonary hypertension (CTEPH) is a rare disease estimated to result in approximately $3.8 \%$ of all cases of pulmonary embolism [1] . It has emerged as one of the leading causes of severe pulmonary hypertension. (CTEPH) is defined as symptomatic pulmonary hypertension (mean PAP: $25 \mathrm{mmHg}$ ) with persistent lung perfusion defects [2].

The disease is caused by chronic obstruction of pulmonary artery branches following episodes of pulmonary embolism and incomplete thrombus resolution leading to intraluminal fibrous stenosis [3].CTPA has become the method of choice for the diagnosis of PE in clinical practice [4].

The goal of pulmonary thromboendarterectomy is to decrease the extent of pulmonary vascular obstruction, thereby reducing pulmonary arterial pressure and improving cardiac function. Surgical outcomes with respect to quality of life, functional status, hemodynamics, right ventricular function and gas exchange are favourable [5].

Before anaesthesia, chronic medical treatment already being administered for pulmonary hypertension and right-sided heart failure should be continued. Induction of anaesthesia should be done very cautiously as there is a risk of sudden hemodynamic collapse $[6,7]$.

The pulmonary endarterectomy procedure is performed during total circulatory arrest under conditions of profound hypothermia $\left(18-20^{\circ} \mathrm{C}\right)$. This is required to enable visibility in the distal pulmonary arterial branches, which would otherwise be subject to back-bleeding during the endarterectomy due to the development of a systemic-to-pulmonary artery circulation at the precapillary level [8].

In the majority of patients, the postoperative course is characterized by a marked increase in cardiac output, with a concomitant decrease in pulmonary artery pressures and pulmonary vascular resistance (PVR) both immediate and sustained. Patients are generally mechanically ventilated for at least $24 \mathrm{~h}$ with a maximal inspiratory pressure maintained below $30 \mathrm{~cm}$ of water. The fio 2 level is kept as low as possible ensuring an oxygen saturation of over $90 \%$, and the haematocrit is kept high $(30-32 \%)[9,10]$.

Investigators have tried to define the predictors of operative mortality of which the most important were severe pulmonary hypertension (PA systolic pressure greater than 50 $\mathrm{mmHg}$, and a reduction in PVR of less than $50 \%$ after surgery [11].

Anticoagulation for postoperative venous thrombosis and re-occlusion prophylaxis is started within 4-8 h after surgery. A life-long anticoagulation is mandatory to prevent reocclusion [12]. 
Besides the late complications like sepsis, acute renal failure and cerebrovascular accident a specific complication that occurs in most of the cases is the reperfusion pulmonary edema also known as the reperfusion syndrome [13,14].

Reperfusion injury is defined as a radiologic opacity seen in the lungs within $72 \mathrm{hrs}$ of PEA [15]. It occurs soon after the operation, is associated with profound desaturation , and adversely affects the patients clinical course. The underlying mechanism is thought to be the sudden onset of blood flow to the lung tissue which has not been exposed to the raised pulmonary vascular pressure [16].

In our case there was severe reperfusion pulmonary edema resulting in difficult weaning from mechanical ventilation. This was managed by continuing elective mechanical ventilation, till the hemodynamic and ventilatory parameters were stabilized. Aggressive treatment with diuretics, oral warfarin and sildenafil citrate and monitoring the hemodynamic and ventilatory parameters, acid base study, serial chest x-rays and echocardiography were the mainstay in postoperative treatment.

CONCLUSIONS: Pulmonary endarterectomy is an effective and potentially curative surgical treatment for patients with severe CTEPH. Medical therapy for this disease is palliative, and ineffective in prolonging life. Reperfusion pulmonary edema is an early postoperative complication which can be dealt with efficient and aggressive management to reduce postoperative morbidity. Adequate preoperative patient evaluation, selection and pretreatment, surgical and anaesthetic technique, meticulous postoperative management, and a multidisciplinary approach are the basic pre-requisites for a successful PEA.

\section{REFERENCES :}

1. 1.Pengo V, Lensing A W, Prins $\mathrm{MH}$, Marchiori A, Davidson BL, et al. Incidence of thromboembolic pulmonary hypertension after pulmonary embolism. New England Journal of Medicine,2004;350: 2257-64.

2. E.Mayer. Surgical and post-operative treatment of chronic thomboembolic pulmonary hypertension . Eur Respi Rev. 2010;19:115,64-67.

3. Mayer E, Klepetko W. Techniques and outcomes of pulmonary endarterectomy for chronic thromboembolic pulmonary hypertension. Proc Am Thorac Soc 2006; 3: 589593.

4. 4Schoepf UJ, Goldhaber SZ, Costello P. Spiral computed tomography for acute pulmonary embolism. Circulation 2004;109:2160-7.

5. William R. Auger, Kim M. Kerr, Nick H.S. Kim, Chronic thromboembolic pulmonary hypertension. Cardiol Clin 22 (2004) 453 -466.

6. Peter Rosenberger, Stanton K. Shernan, Prem S. Shekar, et al. Acute Hemodynamic Collapse After Induction of General Anesthesia for Emergent Pulmonary Embolectomy (Anesth Analg 2006;102:1311-5).

7. Kitamura T, Morota T, Takamoto S. Emergent pulmonary thromboendarterectomy with percutaneous cardiopulmonary support system for chronic thromboembolic pulmonary hypertension. Eur J Cardiothorac Surg 2003; 24:656-658.

8. Dartevelle P, Fadel E, Mussot S, Chapelier A, et al. Chronic thromboembolic pulmonary hypertension. Eur Respir J 2004;23:637-648. 
9. Fedullo PF, Auger WR, Dembitsky WP. Postoperative management of the patient undergoing pulmonary thromboendarterectomy. Semin Thorac Cardiovasc Surg 1999; 11:172-178.

10. D'Armini AM, Cattadori B, Monterosso C, et al. Pulmonary thromboendarterectomy in patients with chronic thromboembolic pulmonary hypertension: hemodynamic characteristics and changes. Eur J Cardiothorac Surg 2000; 18:696-702.

11. Hartz RS, Byme JG, Levitsky S, Park J, Rich S. Predictors of mortality in pulmonary thromboendarterectomy. Ann Thorac Surg 1996;62:1255-9.

12. 12.Roland Demeyerea, Marion Delcroixb and Willem Daenen. Anaesthesia management for pulmonary endarterectomy .Current Opinion in Anaesthesiology 2005, 18:63-76.

13. 13.Thistlethwaite PA, Kaneko K, Madani MM,Jamieson SW.Technique and outcomes of pulmonary endarterectomy surgery. Ann Thorac cardiovasc surgery 2008; 14: 274-82.

14. Jamieson SW, Kapelanski DP, Sakakibara N, Manecke GR, Thistlethwaite PA, Kerr KM, et al. Pulmonary endarterectomy: experience and lessons learned in 1,500 cases. Ann Thorac Surg 2003;76(5):1457-64.

15. Lee K-C, Cho Y-L, Lee S-Y. Reperfusion pulmonary edema after pulmonary thromboendarterectomy. Acta Anaesth Sin 2001; 39:97-101.

16. Pepke-Zaba J, Jamieson SW, Lang I, Corris P. Interventional and surgical modalities of treatment for pulmonary arterial hypertension. J Am Coll Cardiol. 2004;43(suppl S):73S- 80S.

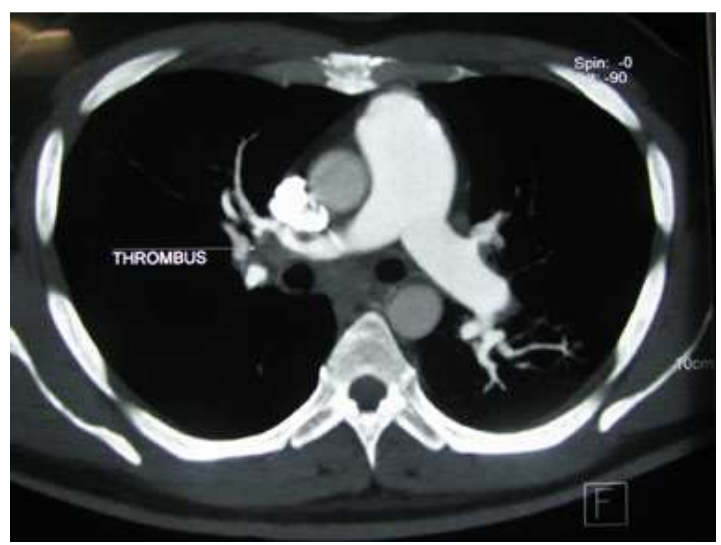

Figure 1.CT image of Pulmonary Embolus

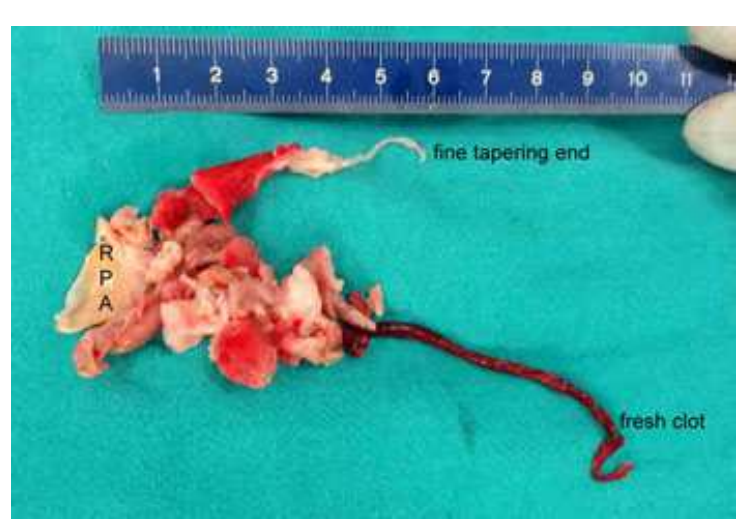

Figure 2. Endarterectomy specimen 